\title{
Análisis multicéntrico del reparo de la hernia ventral en instituciones de IV nivel, 2015-2019
}

\author{
Multicenter analysis of ventral hernia repair in IV level institutions, \\ 2015-2019 \\ Cristina Judith Padilla', Neil Valentín Vega² ${ }^{2}$ Arnold José Barrios ${ }^{3}$, Juan Pablo Ruiz , \\ Alejandro Lora ${ }^{4}$
}

\begin{abstract}
Médica, residente de Cirugía General, Fundación Universitaria Sanitas, Bogotá, D.C., Colombia Médico, cirujano general, FACS, Departamento de Cirugía, Clínica Reina Sofía, Bogotá, D.C., Colombia

Médico, cirujano general, FACS; director, Departamento Quirúrgico de Clínicas Colsanitas, Clínica Reina Sofía, Bogotá, D.C., Colombia

4 Médico, cirujano general, Departamento de Cirugía, Clínica Universitaria Colombia y Clínica Reina Sofia, Bogotá, D.C., Colombia Segundo puesto, Concurso Nacional del Residente Quirúrgico, 45 Congreso Semana Quirúrgica Nacional, agosto de 2019, Bogotá, D.C., Colombia
\end{abstract}

\section{Resumen}

Introducción. La cirugía de hernia ventral implica una situación de complejidad, dadas las múltiples variables que se deben controlar para estimar los posibles factores determinantes del éxito quirúrgico y la aparición de complicaciones. Según la literatura científica mundial, la incidencia de hernia ventral se estima entre el ro y el I5 \%, y la tasa promedio de complicaciones de esta cirugía varía entre el io y el $37 \%$.

El objetivo del presente estudio fue describir la experiencia y los resultados de la cirugía de hernia ventral en dos instituciones de IV nivel, en el periodo de enero de 2015 a marzo de 2019.

Métodos. Se trata de un estudio observacional, descriptivo y de cohorte histórica, de pacientes mayores de edad sometidos a corrección de hernia ventral en las Clínicas Colsanitas en los últimos cinco años. Los datos se tomaron del registro estadístico de las instituciones en mención.

Resultados. Se incluyeron 6 I2 pacientes en un periodo de cinco años, la mayoría de los cuales era de sexo femenino, con sobrepeso, y predominantemente, con defectos combinados mediales; la tasa general de complicaciones fue del $20 \%$ y, el porcentaje de infección del sitio operatorio, de $9 \%$; para el desarrollo de esta infección, la técnica de separación de componentes se encontró como un factor de riesgo ( $\left.\mathrm{p}=\mathrm{O}, \mathrm{OI} ; \mathrm{RR}=2,9 ; \mathrm{IC}_{95 \%} \mathrm{I}, 32-6,5\right)$. En este estudio, no se analizó la recidiva como factor de los diferentes resultados.

Fecha de recibido: 30/06/2019 - Fecha aceptación: 21/08/2019

Correspondencia: Cristina Padilla, Calle 45 N 45-16, Bogotá, D.C., Colombia, Teléfono: (301) 658-5046

Correo electrónico: judithpa2014@gmail.com

Citar como: Padilla CJ, Vega NV, Barrios AJ, Ruiz JP, Lora A. Análisis multicéntrico del reparo de la hernia ventral en instituciones de IV nivel, 2015-2019. Rev Colomb Cir. 2020;35:43-50. https://doi.org/10.30944/20117582.587

Este es un artículo de acceso abierto bajo una Licencia Creative Commons - BY-NC-ND. https://creativecommons.org/licenses/bync-nd/4.0/deed.es 
Conclusiones. Existen pocos datos en la literatura nacional sobre los resultados de este tipo de procedimiento quirúrgico. Es por ello que se procuró brindar a la comunidad científica los resultados de morbimortalidad de esta muestra de pacientes intervenidos por hernia ventral en los últimos cinco años.

Palabras clave: hernia ventral; hernia incisional; reconstrucción de pared abdominal; mallas quirúrgicas; prótesis e implantes; infección de la herida quirúrgica.

\begin{abstract}
Introduction: Ventral hernia surgery involves a complex scenario, given the multiple variables that must be controlled to estimate the possible determinants of surgical success and the appearance of complications. According to the world literature, the incidence of ventral hernia is estimated between $10 \%$ and $15 \%$, and the average complication rate of this surgery varies between $10 \%$ and $37 \%$.
\end{abstract}

The objective of this study was to describe the experience and outcomes in ventral hernia surgery in two institutions of IV level, in the period from January 2015 to March 2019.

Methods: This is an observational, descriptive and historical cohort study of patients undergoing correction of ventral hernia at Colsanitas Clinics in the last five years. The data were collected from the statistical record of the mentioned institutions.

Results: A total of 612 patients were included in a five-year period, most of whom were female, overweight, and predominantly with medial combined defects; the overall complication rate was $20 \%$, and the percentage of operative site infection, 9\%. From the development of this infection, the component separation was found as a risk factor ( $\mathrm{p}=0.0 \mathrm{O} ; \mathrm{RR}=2.9 ; \mathrm{CI}_{95 \%} \mathrm{I} .32-6.5$ ). In this study, recurrence was not analyzed as an outcome factor.

Conclusions: There is little data in the national literature on the results of this type of surgical procedure, which is why we tried to provide the scientific community with the morbidity and mortality results in our population of patients operated for ventral hernia in the last five years.

Key words: hernia, ventral; incisional hernia; abdominal wall reconstruction; surgical mesh; prostheses and implants; surgical wound infection.

\section{Introducción}

La hernia ventral se define como una protrusión anormal del contenido de la cavidad abdominal o de la grasa preperitoneal a través de un defecto o debilidad que se desarrolla usualmente después de un procedimiento quirúrgico ${ }^{\mathrm{I}}$.

Este padecimiento es una complicación común de la cirugía abdominal, con una incidencia que varía entre el io y el I5\%, y se incrementa hasta el $80 \%$ en los pacientes que desarrollan infección de la herida ${ }^{2}$. Se ha informado que estos defectos aparecen como resultado de una alteración molecular en la que se altera la cicatrización, principalmente, por disminución del colágeno de tipo I y de tipo 3, así como por un defecto en las metaloproteasas de matriz. Además de lo anterior, se describen ciertos factores de riesgo que se cree que pueden aumentar la probabilidad de aparición de estas hernias, como son el estado nutricional, la inmunosupresión y el tabaquismo, entre otras ${ }^{3}$.

En cuanto al tipo de defecto, según la clasificación de la European Hernia Society (EHS), las hernias de la pared abdominal se dividen, según su origen, en primarias y adquiridas; a su vez, estas últimas se subdividen según su localización, en mediales y laterales (figura I) y, según su tamaño, en tres categorías; los defectos mayores de Io $\mathrm{cm}$ se consideran hernias gigantes ${ }^{4}$. 


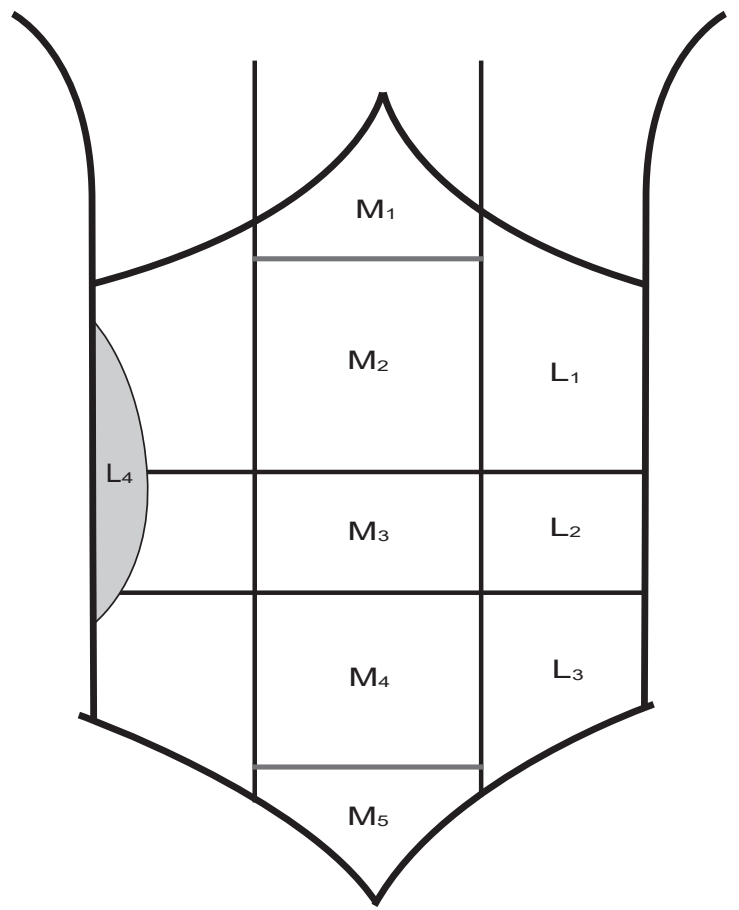

Figura 1. Esquema grafico de la clasificación de las hernias por incisión de la pared abdominal. $\mathrm{M}_{1}$ defecto medial subxifoideo; $M_{2}$ defecto medial epigástrico; $M_{3}$ defecto medial umbilical; $M_{4}$ defecto medial infraumbilical; $M_{5}$ defecto medial suprapúbico; $L_{1}$ defecto lateral subcostal; $L_{2}$ defecto lateral flanco; $L_{3}$ defecto lateral iliaco; $L_{4}$ defecto lateral lumbar

En el pasado, el objetivo de la reparación de las hernias ventrales consistía en aproximar los bordes de la fascia con una sutura continua o interrumpida, y la tasa de recurrencia a largo plazo era superior al $63 \%^{3}$. El aprendizaje sobre la etiología y la dinámica de la pared abdominal hizo que, desde los años 50, se empezaran a publicar los primeros escritos sobre las técnicas sin tensión, entre las que se destacan la primera malla de polipropileno y, posteriormente, en los 70, técnicas como la de Rives-Stoppa ${ }^{5,6}$.

El impacto favorable de la disminución de recidivas es evidente tras la utilización de prótesis en la cirugía de la hernia ventral, aun cuando su número continúa siendo elevado, con un porcentaje que oscila entre el 2 y el $36 \%$ de los casos ${ }^{7}$.

La cirugía de la hernia ventral implica una situación compleja, dadas las múltiples variables que se deben controlar, entre ellas la aparición de complicaciones, principalmente con la técnica abierta, con un rango de o a $33 \%$, y con una proporción del 3,8 \% para la técnica laparoscópica ${ }^{6,8}$. Algunos de los factores descritos en relación con la aparición de complicaciones, son el tipo de técnica quirúrgica utilizada, las condiciones propias del paciente (diabetes, obesidad, tabaquismo, etc.), el uso de mallas y el tiempo operatorio, entre otros ${ }^{\text {9,Io }}$.

Entre las complicaciones posoperatorias de la corrección quirúrgica de la hernia ventral, están la infección del sitio operatorio, que varía del 4 al $5 \%$, y los eventos del sitio operatorio (seroma, hematoma y granuloma, entre otros), de 5,5\% con la técnica abierta y de I,2 \% con la laparoscópica" ${ }^{\mathrm{II}}$. En nuestro medio, según Briceño-Morales, en un hospital de II nivel de la capital, se registró una frecuencia de infección del sitio operatorio de $\mathrm{II}, 7 \%$ y una recidiva herniaria de $\mathrm{I} 7,5 \%$, a los Io años de seguimiento 5 .

La aparición de complicaciones del sitio operatorio aumenta tres veces la probabilidad de recidiva de la hernia y, por tanto, las tasas de rehospitalización ${ }^{12}$.

Anualmente, solo en Estados Unidos se realizan cerca de 500.000 reparaciones de hernia ventral, con un costo estimado de USD \$ 3,2 billones ${ }^{13}$ con una tasa de readmisión posterior a la corrección de la hernia ventral, que varía del 5 al I3 \%, con un costo estimado, para el 2007, en Estados Unidos, de USD\$ 15 billones ${ }^{14}$.

En Clínicas Colsanitas de Bogotá, se intervienen anualmente I5O pacientes por hernia ventral, con cirugías que varían en complejidad y presentación. Sin embargo, existen pocos datos institucionales informados en el país sobre los resultados de este tipo de procedimiento quirúrgico. Por esto, la relevancia del presente estudio se sustentó en la necesidad de generar evidencia científica derivada de la experiencia institucional alrededor del tema.

El objetivo de esta publicación fue describir la experiencia y los resultados de la reparación quirúrgica de la hernia ventral en dos instituciones de IV nivel de Bogotá, en el periodo de enero de 2015 a marzo de 2019. 


\section{Métodos}

Se llevó a cabo un estudio observacional retrospectivo, analítico, de cohorte y con un muestreo no probabilístico, del universo de pacientes intervenidos quirúrgicamente por hernia ventral entre enero de 2015 y marzo de 2019 en dos clínicas de IV nivel de Bogotá. Se incluyeron pacientes mayores de I8 años intervenidos por hernia ventral mediante cualquier técnica. Se excluyeron los pacientes menores de edad y aquellos con historias clínicas incompletas.

Las hernias se clasificaron de acuerdo con la escala de la EHS, como defectos mediales, laterales o combinados (figura I). Las variables que se evaluaron fueron, principalmente, las relacionadas con los resultados quirúrgicos, como: características demográficas, tipo de técnica quirúrgica, posición de las prótesis, tiempo quirúrgico y tipo de fijación utilizada, entre otras. Se clasificaron los resultados según lo establecido por los Centers for Disease Control and Prevention (CDC) de Atlanta, en superficial, profunda y de órgano-espacio, y la clasificación del grupo de trabajo de hernia ventral de eventos del sitio operatorio, como seroma, hematoma, dehiscencia, etc. ${ }^{15,16}$.

\section{Fuente de información}

Los datos se recolectaron del registro estadístico de las instituciones, valorando las historias clínicas que se encontraron en formato digital en el sistema SOPHIA de los pacientes intervenidos en el periodo de estudio.

\section{Análisis estadístico}

Las variables cuantitativas se analizaron mediante medidas de frecuencia, tendencia central y dispersión, y los datos categóricos, mediante proporciones. Los supuestos de distribución normal de las variables de interés se evaluaron con el test no paramétrico de Shapiro-Wilk y mediante análisis gráficos (histogramas de frecuencias, box plot y gráficos Q-Q). Los análisis bivariados y de correlación con la prueba de ji al cuadrado $\left(\chi^{2}\right)$ para independencia y homogeneidad entre variables, en tablas de $2 \times 2$. Según la naturaleza de la distribución de las variables continuas, se usó la prueba estadística t de Student. Los datos se analizaron con el software Stata 13.0 ${ }^{\mathrm{TM}}$ (http://www.stata.com).

\section{Resultados}

Se analizaron 612 pacientes intervenidos por hernia ventral por el Grupo de Pared Abdominal en la Clínica Reina Sofía y en la Clínica Universitaria Colombia, de enero de 2015 a marzo del 20I9. El 5I \% ( $\mathrm{n}=353)$ de los pacientes era de sexo femenino, con un promedio de edad de 58,3 años (desviación estándar, DE: \pm I5,2 años) (tabla I).

El índice de masa corporal promedio de la muestra fue de $27,8 \mathrm{~kg} / \mathrm{m}^{2}$ (DE: $\pm 4,6 \mathrm{~kg}$ ); el 79,7\% $(\mathrm{n}=488)$ presentó alguna comorbilidad asociada, siendo la de mayor prevalencia el tabaquismo (22\%), seguida de diabetes mellitus y cáncer en el I2 \%. Los defectos ventrales más comunes fueron los medianos combinados en el $40 \%$ ( $n=240)$, seguido de los defectos umbilicales (M3) en el $9 \%(n=5 I)$, $y$ los defectos mediales y laterales combinados en $8 \%(\mathrm{n}=45)$.

En el $64 \%(n=379)$ de los pacientes intervenidos, se usó la técnica abierta, en $24 \%$ ( $n=I 43)$, la laparoscópica y, en I2 \% ( $\mathrm{n}=7 \mathrm{I})$, la combinada. El tiempo quirúrgico promedio fue de 88,6 minutos; el tipo de material protésico más utilizado fue el de mediana densidad, en el $58 \%$ ( $n=343)$, y en menos del I \%, se colocó la malla inlay. Además, del total de las intervenciones, en el I,7 \% (n=II) se utilizó el neumoperitoneo preoperatorio (tabla 2).

La mediana de la estancia hospitalaria fue de 4 días (rango intercuartílico, $\mathrm{RIC}=3$ días). Solo en el $3 \%$ se desarrolló infección del sitio operatorio, con mayor incidencia de la superficial ( $4 \%)$ y, más frecuentemente, entre el $9^{\circ}$ y el $5^{\circ}$ día posoperatorios (tabla 3).

Se produjeron 17 reingresos, la mayoría de ellos por cuadros de obstrucción intestinal. La mortalidad registrada fue de $0,4 \%$, principalmente por sepsis de origen abdominal, y solo uno de los pacientes falleció a consecuencia de una infección respiratoria aguda.

En el análisis bivariado se encontró que la aparición de infecciones del sitio operatorio también tenía una asociación significativa con la posición supraponeurótica (onlay) de la malla $(\mathrm{p}=0,002)$, a diferencia de variables como la técnica quirúrgica, el tabaquismo y la diabetes, en las que no se encontraron diferencias significativas en la aparición de complicaciones a los 30 días del seguimiento (tabla 4). 
Tabla 1. Características de los pacientes intervenidos por cirugía de hernia ventral en Clinicas Colsanitas, Bogotá, D.C., Colombia

\begin{tabular}{lcc}
\hline \multicolumn{1}{c}{ Variable } & $\mathbf{n}$ & $\%$ \\
\hline Sexo & 353 & 51 \\
Femenino & 241 & 49 \\
Masculino & & \\
Edad (años) & 58,5 & \\
$\quad$ Mujeres (promedio) & 57,9 & \\
Hombres (promedio) & $27,8 \mathrm{~kg}$ & \\
Índice de masa corporal (promedio) & & \\
Antecedentes personales & 132 & 22 \\
Tabaquismo & 71 & 12 \\
Diabetes mellitus & 5 & 0,8 \\
Artritis & 1 & 0,2 \\
Lupus eritematoso sistémico & 69 & 12 \\
Cáncer & 106 & 18 \\
Reintervención quirúrgica & & \\
Clasificación ASA & 90 & 15 \\
Sin clasificación & 112 & 19 \\
I & 295 & 50 \\
II & 95 & 16 \\
III & 2 & 0,3 \\
IV & & \\
Estancia hospitalaria & 4 & \\
Días (promedio) & 17 & 2,77 \\
Reingresos & 3 & 0,49 \\
Mortalidad & &
\end{tabular}

Además, dicho análisis puso en evidencia que la posición de la malla influye de forma estadísticamente significativa en la aparición de eventos del sitio operatorio $(\mathrm{p}=0,037)$ : posición supraaponeurótica (onlay) y desarrollo de seroma, $R R=2,4$ e $\mathrm{IC}_{95} \%$ I,08-5,6; y posición infraaponeurótica (sublay) y desarrollo de seroma, RR=0,59 e IC ${ }_{95 \%}$ 0,2-I,3 (tabla 5).

Entre los procedimientos por técnica abierta, el $28 \%$ (n=I66) correspondía a la de separación de componentes y, en el análisis bivariado, se encontró una relación estadísticamente significativa entre este procedimiento y el desarrollo de infección del sitio operatorio ( $\mathrm{p}=\mathrm{O}, \mathrm{OI})$, específicamente, para la profunda $\left(\mathrm{RR}=4,6 ; \mathrm{IC}_{95} \% \mathrm{I}, \mathrm{II}-\mathrm{I} 9\right)$.

\section{Discusión}

La cirugía abdominal es uno de los procedimientos quirúrgicos más comunes a nivel mundial $y$, entre sus complicaciones, se encuentran las hernias ventrales, con una incidencia que puede
Tabla 2. Procedimientos quirúrgicos practicados a los pacientes con hernia ventral en Clínicas Colsanitas, Bogotá, D.C., Colombia

\begin{tabular}{|c|c|c|}
\hline Variable & $\mathbf{n}$ & $\%$ \\
\hline \multicolumn{3}{|l|}{ Técnica quirúrgica } \\
\hline Abierta & 379 & 64 \\
\hline Laparoscópica & 143 & 24 \\
\hline Combinada (mixta) & 71 & 12 \\
\hline \multicolumn{3}{|l|}{ Tipo de hernia ventral } \\
\hline$M_{1}$ & 28 & 5 \\
\hline $\mathrm{M}_{2}$ & 40 & 7 \\
\hline$M_{3}$ & 51 & 9 \\
\hline $\mathrm{M}_{4}$ & 16 & 3 \\
\hline $\mathrm{M}_{5}$ & 8 & 1 \\
\hline $\mathrm{L}_{1}$ & 12 & 2 \\
\hline $\mathrm{L}_{2}$ & 20 & 3 \\
\hline $\mathrm{L}_{3}$ & 37 & 6 \\
\hline $\mathrm{L}_{4}$ & 4 & 1 \\
\hline Combinadas mediales & 240 & 40 \\
\hline Combinadas laterales & 29 & 5 \\
\hline $\begin{array}{l}\text { Combinadas mediales y } \\
\text { laterales }\end{array}$ & 45 & 8 \\
\hline \multicolumn{3}{|l|}{ Tipo de material protésico } \\
\hline Baja densidad & 141 & 24 \\
\hline Mediana densidad & 343 & 58 \\
\hline Alta densidad & 31 & 5 \\
\hline \multicolumn{3}{|l|}{ Posición de la malla } \\
\hline Onlay & 113 & 22 \\
\hline Inlay & 3 & 0,6 \\
\hline Sublay & 273 & 54 \\
\hline Intraperitoneal & 85 & 17 \\
\hline Sándwich & 36 & 7 \\
\hline \multicolumn{3}{|l|}{ Tipo de fijación } \\
\hline Absorbible & 38 & 6 \\
\hline No absorbible & 99 & 17 \\
\hline Mixta & 241 & 41 \\
\hline Sutura & 102 & 17 \\
\hline Pegamento & 55 & 9 \\
\hline \multicolumn{3}{|l|}{ Separación de componentes } \\
\hline Sí & 166 & 28 \\
\hline \multicolumn{3}{|l|}{ Tiempo quirúrgico (minutos) } \\
\hline Abierta (promedio) & 148 & \\
\hline Laparoscópica (promedio) & 104 & \\
\hline Combinada (mixta) (promedio) & 147 & \\
\hline \multicolumn{3}{|l|}{ Sangrado intraoperatorio (ml) } \\
\hline Abierta (promedio) & 55 & \\
\hline Laparoscópica (promedio) & 11 & \\
\hline Combinada (mixta) (promedio) & 23 & \\
\hline
\end{tabular}


Tabla 3. Complicaciones posoperatorias de los pacientes intervenidos por cirugía de hernia ventral en Clinicas Colsanitas, Bogotá, D.C., Colombia

\begin{tabular}{lcc}
\hline & $\mathbf{n}$ & $\%$ \\
\hline $\begin{array}{l}\text { Infección del sitio operatorio } \\
\text { Superficial }\end{array}$ & 23 & 4 \\
Profunda & 8 & 1,4 \\
Órgano-espacio & 7 & 1,2 \\
Tiempo de ISO (días) & & \\
$\leq 8$ & 20 & 39 \\
$\leq 5$ & 26 & 51 \\
30 & 5 & 10 \\
Eventos del sitio operatorio & & \\
Seroma & 23 & 4 \\
Dehiscencia & 19 & 3 \\
Hematoma & 14 & 2 \\
Granuloma & 2 & 0,3 \\
Fistula & 3 & 0,5 \\
Varios & 3 & 0,5 \\
Otros (perforación intestinal) & 1 & 0,2 \\
Tiempo de ESO (días) & & \\
$\leq 8$ & 21 & 38 \\
$\leq 15$ & 23 & 41 \\
30 & 12 & 1 \\
\hline
\end{tabular}

ISO: infección del sitio operatorio; ESO: eventos del sitio operatorio
Tabla 4. Factores de riesgo para el desarrollo de infección del sitio operatorio en los pacientes intervenidos por cirugía de hernia ventral en Clínicas Colsanitas, Bogotá, D.C., Colombia

\begin{tabular}{|c|c|c|c|c|}
\hline \multicolumn{5}{|c|}{ Infección de sitio operatorio } \\
\hline Factor de riesgo & $\begin{array}{c}\text { Superficial } \\
\text { n (\%) }\end{array}$ & $\begin{array}{c}\text { Profunda } \\
\text { n (\%) }\end{array}$ & $\begin{array}{c}\text { Órganol } \\
\text { espacio } \\
\text { n (\%) }\end{array}$ & $\underset{I C_{95 \%}}{p}$ \\
\hline Diabetes mellitus & $2(2,8)$ & $0(0)$ & $1(1,4)$ & 0,8 \\
\hline Tabaquismo & $4(3)$ & $2(1,5)$ & $2(1,5)$ & 0,13 \\
\hline \multicolumn{5}{|l|}{ Técnica quirúrgica } \\
\hline $\begin{array}{l}\text { Abierta } \\
\text { Laparoscópica } \\
\text { Mixta }\end{array}$ & $\begin{array}{l}18(4,7) \\
4(2,8) \\
1(1,4)\end{array}$ & $\begin{array}{l}8(2,1) \\
0(0) \\
0(0)\end{array}$ & $\begin{array}{l}6(1,5) \\
1(0,7) \\
0(0)\end{array}$ & 0,78 \\
\hline \multicolumn{5}{|c|}{ Posición de la prótesis* } \\
\hline $\begin{array}{l}\text { Onlay }{ }^{1} \\
\text { Inlay }^{2} \\
\text { Sublay } \\
\text { Intraperitoneal } \\
\text { Doble malla } \\
\text { (técnica sándwich) }\end{array}$ & $\begin{array}{c}7(6,1) \\
0(0) \\
7(2,5) \\
2(2,3) \\
5(13,8)\end{array}$ & $\begin{array}{c}2(1,7) \\
0(0) \\
2(0,7) \\
1(1,18) \\
3(8,3)\end{array}$ & $\begin{array}{c}1(0,8) \\
0(0) \\
1(0,3) \\
1(1,18) \\
1(2,7)\end{array}$ & 0,002 \\
\hline $\begin{array}{l}\text { Separación de } \\
\text { componentes }\end{array}$ & $12(7,23)$ & $5(3)$ & $3(1,8)$ & 0,017 \\
\hline
\end{tabular}

* 1: supraaponeurótica; ${ }^{2}$ : malla en puente; ${ }^{3}$ : infraaponeurótica

Tabla 5. Factores de riesgo para el desarrollo de eventos del sitio operatorio en los pacientes intervenidos por cirugía de hernia ventral en Clínicas Colsanitas, Bogotá, D.C., Colombia

\begin{tabular}{|c|c|c|c|c|c|c|c|c|}
\hline \multicolumn{9}{|c|}{ Eventos del sitio operatorio } \\
\hline Factor de riesgo & $\begin{array}{l}\text { Seroma } \\
\mathrm{n}(\%)\end{array}$ & $\begin{array}{l}\text { Dehiscencia } \\
\text { n (\%) }\end{array}$ & $\begin{array}{c}\text { Hematoma } \\
\mathrm{n}(\%)\end{array}$ & $\begin{array}{c}\text { Granuloma } \\
\mathrm{n}(\%)\end{array}$ & $\begin{array}{l}\text { Fistula } \\
\text { n (\%) }\end{array}$ & $\begin{array}{l}\text { Varios } \\
\mathrm{n}(\%)\end{array}$ & $\begin{array}{l}\text { Perforación } \\
\text { n (\%) }\end{array}$ & IC $_{95 \%}^{\mathbf{p}}$ \\
\hline Diabetes mellitus & $4(5,6)$ & $3(4,2)$ & $0(0)$ & $0(0)$ & $0(0)$ & $0(0)$ & $0(0)$ & 0,12 \\
\hline Tabaquismo & $5(3,7)$ & $7(5,3)$ & $4(3)$ & $1(0,76)$ & $1(0,76)$ & $0(0)$ & $0(0)$ & 0,65 \\
\hline \multicolumn{9}{|l|}{ Técnica quirúrgica } \\
\hline Abierta & $18(4,7)$ & $16(4,2)$ & $10(2,6)$ & $2(0,5)$ & $1(0,2)$ & $3(0,7)$ & $0(0)$ & \\
\hline Laparoscópica & $4(2,8)$ & $1(0,7)$ & $4(2,8)$ & $0(0)$ & $1(0,7)$ & $0(0)$ & $1(0,7)$ & \\
\hline Mixta & $1(1,4)$ & $2(2,8)$ & $0(0)$ & $0(0)$ & $1(1,4)$ & $0(0)$ & $0(0)$ & 0,73 \\
\hline \multicolumn{9}{|c|}{ Posición de la prótesis } \\
\hline Onlay & $9(7,9)$ & $4(3,5)$ & $4(3,5)$ & $0(0)$ & $0(0)$ & $1(0,8)$ & $0(0)$ & \\
\hline Inlay & $0(0)$ & & $0(0)$ & $0(0)$ & $0(0)$ & $0(0)$ & $0(0)$ & \\
\hline Sublay & $9(3,3)$ & $6(2,2)$ & $8(2,9)$ & $1(0,3)$ & $0(0)$ & $2(0,7)$ & $0(0)$ & \\
\hline Intraperitoneal & $1(1,1)$ & $0(0)$ & $0(0)$ & $0(0)$ & $1(1,1)$ & $0(0)$ & $1(1,1)$ & 0,01 \\
\hline $\begin{array}{l}\text { Doble malla (técnica } \\
\text { sándwich) }\end{array}$ & $3(8,3)$ & $4(11,1)$ & $1(2,7)$ & $0(0)$ & $1(2,7)$ & $0(0)$ & $0(0)$ & \\
\hline $\begin{array}{l}\text { Separación de } \\
\text { componentes }\end{array}$ & $11(6,6)$ & $9(5,4)$ & $4(2,4)$ & $1(0,6)$ & $0(0)$ & $2(1,2)$ & $0(0)$ & 0,07 \\
\hline
\end{tabular}


alcanzar hasta el I5,2 \%, como lo describen Cherla, et al., en un artículo observacional publicado en $2018^{17}$.

$\mathrm{Al}$ igual que Briceño-Morales, en el presente estudio se encontró que estas hernias fueron más frecuentes en el sexo femenino, con un promedio de edad de 58 años 5 . Diferentes publicaciones, entre las que se encuentra la de Kaoutzanis, et al., describen la asociación entre el tabaquismo, un IMC mayor de $30 \mathrm{~kg} / \mathrm{m}^{2}$ y una clasificación de ASA de 3 o más, y la aparición de complicaciones de la herida quirúrgica. Sin embargo, en el presente estudio, a pesar de que el tabaquismo y la diabetes fueron las comorbilidades más frecuentes, en el análisis univariado no se encontró significación estadística en la relación entre dichos factores y la infección u otros eventos del sitio operatorio ${ }^{2}$.

Tradicionalmente, las hernias suelen corregirse por vía abierta. Sin embargo, con el advenimiento de la cirugía mínimamente invasiva y sus ventajas, como la menor estancia hospitalaria y la menor tasa de complicaciones de la herida, en la actualidad, son cada vez más frecuentes las herniorrafias por laparoscopia; así lo demostraron Kokotovic, et al., en un estudio retrospectivo de cohorte, en el que el $54,2 \%$ de los pacientes con hernia ventral fueron intervenidos mediante laparoscopia ${ }^{18}$.

A pesar de lo anterior, en un estudio prospectivo de cinco años, que incluyó 710 pacientes, se registró que solo el $27,4 \%$ de las hernias ventrales se reparan por vía laparoscópica, dato que concuerda con lo encontrado en el presente estudio, en el cual el abordaje laparoscópico se utilizó en el $24 \%$ de la población ${ }^{7,8,19}$.

En la técnica abierta se encuentra la separación de componentes, procedimiento que cada vez gana más popularidad para la reparación de hernias complejas, que son aquellas que suelen tener un diámetro superior a $10 \mathrm{~cm}$. En una revisión sistemática del 2017 que incluyó 36 estudios, se encontró que esta técnica se asoció con eventos del sitio operatorio en I6 a $23 \%$ de los casos, con significación estadística en la separación posterior $(\mathrm{p}<\mathrm{O}, \mathrm{OOI}){ }^{20}$. A diferencia de ello, en el presente estudio, el $28 \%$ de los pacientes fueron sometidos a separación de componentes y, en el análisis bivariado, se encontró asociación estadísticamente significativa entre este procedimiento y la infección del sitio operatorio ( $\left.\mathrm{p}=\mathrm{O}, \mathrm{OI} ; \mathrm{RR}=2,23 ; \mathrm{IC}_{95 \%} \mathrm{I}, 36-3,66\right)$; sin embargo, no se encontró asociación entre esta técnica y otros eventos del sitio operatorio $(\mathrm{p}=\mathrm{o}, \mathrm{0} 7)$.

En un estudio publicado por el Mayo Clinic Hospital se menciona que la incidencia exacta de infección de las prótesis es difícil de establecer; sin embargo, se cree que esta oscila entre el o,oor y el $8 \%$ de los pacientes sometidos a este tipo de procedimiento quirúrgico. En el presente estudio, se encontró un porcentaje general de infección del sitio operatorio del $9 \%$, cifra más alta que lo registrado en la literatura ${ }^{21}$.

En Clínicas Colsanitas, en el 58 \% de la población se utilizó prótesis de mediana densidad, y se encontró una asociación estadísticamente significativa entre la posición de la prótesis y la ocurrencia de infección del sitio operatorio ( $\mathrm{p}=\mathrm{0}, 002)$, así como con el desarrollo de eventos del sitio operatorio $(\mathrm{p}=\mathrm{O}, \mathrm{OI})$; el riesgo de desarrollar seroma fue tres veces más alto, principalmente en las mallas supraaponeuróticas (onlay) $\left(\mathrm{RR}=2,47 ; \mathrm{IC}_{95 \%} \mathrm{I}, \mathrm{O} 8-5,63\right)$.

En un estudio retrospectivo de Fortelny, et al., se encontró que del 6 al $20 \%$ de las complicaciones de la cirugía de hernia ventral, se relacionan con el uso de prótesis, y se concluyó que la complicación más frecuente era la obstrucción intestinal ${ }^{22}$; en el presente estudio, también se encontró una tasa general de complicaciones del $20 \%$, siendo la más frecuente la infección superficial del sitio operatorio dentro de los 8 primeros días posoperatorios, seguida del seroma dentro de los 5 primeros días de la intervención quirúrgica. Por otro lado, en esta población de estudio, la condición más prevalente de reingreso fue la obstrucción intestinal, en el 23,5\%.

Para finalizar, el objetivo primordial del presente estudio fue presentar los resultados en los pacientes sometidos a corrección de hernia ventral, en relación con complicaciones desarrolladas en el sitio operatorio, sin analizar las recidivas. Ahora, su principal debilidad es no considerar las recidivas, por cuanto la fortaleza 
de la cohorte es tener los pacientes para efectuar este análisis que, en Colombia, no se ha realizado aún.

La importancia de la publicación del presente estudio radica en el hecho de que existen pocos datos en la literatura nacional sobre los resultados de este tipo de procedimiento quirúrgico. Por ello, al ser el grupo de Clínicas Colsanitas uno de los de mayor experiencia en el manejo de la enfermedad de la pared abdominal, teniendo como referencia una base de datos sólida, se procuró brindar a la comunidad científica los resultados en cuanto a morbimortalidad de nuestra población de pacientes intervenidos por hernia ventral en los últimos cinco años.

\section{Cumplimiento de normas éticas}

Consentimiento informado: Este estudio fue aprobado por el Comité de Ética de las Clínicas Colsanitas. De acuerdo con la Resolución 008430 de 1993 del Ministerio de Salud de Colombia, se consideró que la investigación no tenía riesgo para los pacientes por ser una revisión retrospectiva de historias clínicas, por lo tanto, no se diligenció el consentimiento informado.

Conflicto de intereses: ninguno de los autores reportó conflictos de interés.

Financiación: recursos propios de los autores.

\section{Referencias}

I. González-Mayagoitia JC, Cisneros-Muñoz H, Martínez-Munive A. Guías de práctica clínica para hernias de la pared abdominal. Asoc Mex Hernia. 2015;I-46.

2. Kaoutzanis C, Leichtle SW, Mouawad NJ, Welch KB, Lampman RM, Wahl WL, et al. Risk factors for postoperative wound infections and prolonged hospitalization after ventral/incisional hernia repair. Hernia. 2015;19:II3-23.

3. Jin J, Rosen MJ. Laparoscopic versus open ventral hernia repair. Surg Clin North Am. 2008;88:I083-Ioo.

4. Muysoms FE, Miserez M, Berrevoet F, Campanelli G, Champault GG, Chelala E, et al. Classification of primary and incisional abdominal wall hernias. Hernia. 2009;13:407-I4

5. Briceño-Morales C. Epidemiologia y factores asociados a la recidiva de hernias incisionales de la pared abdominal. Experiencia de io años del Servicio de Cirugía General de un hospital de II nivel de la ciudad de Bogotá, Colombia. Repositorio Universidad Nacional. 2016

6. Mason RJ, Moazzez A, Sohn HJ, Berne TV, Katkhouda $\mathrm{N}$. Laparoscopic versus open anterior abdominal wall hernia repair: 30-day morbidity and mortality using the ACS-NSQIP database. Ann Surg. 20II;254:64I-52.

7. van der Linden FT, van Vroonhoven TJ. Long-term results after surgical correction of incisional hernia. Neth J Surg. 1988;40:127-9.

8. Fligor JE, Lanier ST, Dumanian GA. Current risk stratification systems are not generalizable across surgical technique in midline ventral hernia repair. Plast Reconstruct Surg Glob Open. 2017;5:eI206.

9. Mansmann U, Rieger A, Strahwald B, Crispin A. Risk calculators-methods, development, implementation, and validation. Int J Colorectal Dis. 20I6; 31:IIII-6.

Io. Jones CM, Ashrafian H, Darzi A, Athanasiou T. Guidelines for diagnostic tests and diagnostic accuracy in surgical research. J Invest Surg. 2010;23:57-65.

II. Kaoutzanis C, Leichtle SW, Mouawad NJ, Welch KB, Lampman RM, Cleary RK. Postoperative surgical site infections after ventral/incisional hernia repair: A comparison of open and laparoscopic outcomes. Surg Endosc. 2013;27:222I-30.

I2. Basta MN, Bauder AR, Kovach SJ, Fischer JP. Assessing the predictive accuracy of the American College of Surgeons National Surgical Quality Improvement Project Surgical Risk Calculator in open ventral hernia repair. Am J Surg. 20I6;2I2:272-8I.

13. Cherla DV, Poulose B, Prabhu AS. Epidemiology and Disparities in Care: The Impact of Socioeconomic Status, Gender, and Race on the Presentation, Management, and Outcomes of Patients Undergoing Ventral Hernia Repair. Surg Clin North Am. 20I8;98:43I-4O.

I4. P. A. Baltodano, Y. Webb-Vargas, K. C. Soares, et al. A validated, risk assessment tool for predicting readmission after open ventral hernia repair. Hernia. 20I6;20:II9-29.

15. Garner BH, Anderson DJ. Surgical site infections: An update. Infect Dis Clin North Am. 2016;30:909-929.

I6. Petro CC, O'Rourke CP, Posielski NM, Criss CN, Raigani $\mathrm{S}$, Prabhu AS, et al. Designing a ventral hernia staging system. Hernia. 20I6;20:III-7.

17. Cherla DV, Viso CP, Moses ML, Holihan JL, Ko TC, Kao LS, et al. Clinical assessment, radiographic imaging, and patient self-report for abdominal wall hernias. J Surg Res. 2018;227:28-34.

I8. Kokotovic D, Bisgaard T, Helgstrand F .Long-term recurrence and complications associated with elective incisional hernia repair. JAMA. 2016;316:1575-82.

I9. Colavita PD, Tsirline VB, Belyansky I, Walters AL, Lincourt AE, Heniford BT. Prospective, long-term comparison of quality of life in laparoscopic versus open ventral hernia repair. Ann Surg. 2012;256:714-23.

20. Cornette B, De Bacquer D, Berrevoet F. Component separation technique for giant incisional hernia: A systematic review. Am J Surg . 2018;215:719-26.

2I. Aguilar B, Chapital AB, Madura JA, Harold KL. Conservative management of mesh-site infection in hernia repair. J Laparoendosc Adv Surg Tech A. 20IO;20:249-52.

22. Fortelny RH, Glaser KS, Offner F, Benesch T, Rohr M. Adverse effects of polyvinylidene fluoride-coated polypropylene mesh used for laparoscopic intraperitoneal onlay repair of incisional hernia. $\mathrm{Br} \mathrm{J}$ Surg. 2010;97:II40-5. 